\title{
Combined use of laser Doppler flowmetry and skin thermometry for functional diagnostics of intradermal finger vessels
}

Evgeny A. Zherebtsov

Angelina I. Zherebtsova

Alexander Doronin

Andrey V. Dunaev

Konstantin V. Podmasteryev

Alexander Bykov

Igor Meglinski 


\section{Combined use of laser Doppler flowmetry and skin thermometry for functional diagnostics of intradermal finger vessels}

\author{
Evgeny A. Zherebtsov, ${ }^{a, b, \star}$ Angelina I. Zherebtsova, ${ }^{a}$ \\ Alexander Doronin, ${ }^{c}$ Andrey V. Dunaev, ${ }^{a}$ \\ Konstantin V. Podmasteryev, ${ }^{\mathrm{a}}$ Alexander Bykov, ${ }^{\mathrm{b}, \mathrm{d}, \mathrm{e}}$ \\ and Igor Meglinski $\mathbf{i}^{\mathrm{b}, \mathrm{d}, \mathrm{e}, \mathrm{f}}$ \\ ${ }^{a}$ Orel State University, Scientific-Educational Centre of Biomedical \\ Engineering, Biomedical Photonics Instrumentation Group, Orel, \\ Russia \\ bUniversity of Oulu, ITEE, Optoelectronics and Measurement \\ Techniques Unit, Oulu, Finland \\ 'Yale University, Department of Computer Science, Computer \\ Graphics Group, New Haven, Connecticut, United States \\ ${ }^{\mathrm{d}}$ Tomsk State University, Interdisciplinary Laboratory of Biophotonics, \\ Tomsk, Russia \\ eITMO University, Saint-Petersburg, Russia \\ fIrkutsk State University, Institute of Biology, Irkutsk, Russia
}

\begin{abstract}
We introduce a noninvasive diagnostic approach for functional monitoring of blood microflows in capillaries and thermoregulatory vessels within the skin. The measuring system is based on the combined use of laser Doppler flowmetry and skin contact thermometry. The obtained results suggest that monitoring of blood microcirculation during the occlusion, performed in conjunction with the skin temperature measurements in the thermally stabilized medium, has a great potential for quantitative assessment of angiospatic dysfunctions of the peripheral blood vessels. The indices of blood flow reserve and temperature response were measured and used as the primarily parameters of the functional diagnostics of the peripheral vessels of skin. Utilizing these parameters, a simple phenomenological model has been suggested to identify patients with angiospastic violations in the vascular system. @ 2017 Society of Photo-Optical Instrumentation Engineers (SPIE) [DOI: 10.1117/1.JBO.22.4.040502]
\end{abstract}

Keywords: blood microcirculation; laser Doppler flowmetry; thermometry; occlusion; rheumatology; vasospastic disorders.

Paper 160710LR received Oct. 15, 2016; accepted for publication Mar. 22, 2017; published online Apr. 11, 2017.

\section{Introduction}

Vasospastic disorders are a common class of manifestation of many diseases with different etiology. ${ }^{1,2}$ These include syndromes such as vegetative dystonia, Raynaud's syndrome, vibration disease, rheumatoid arthritis, and others. Diseases of this class are

*Address all correspondence to: Evgeny A. Zherebtsov, E-mail: zherebzow@ gmail.com characterized by prevalence in almost all age groups, steady progression, sporadic manifestation, tendency to chronicity, early disability, and reduced quality of life (especially in case of fingers involvement). ${ }^{1,2}$ In this regard, the development and introduction of new techniques and methods for diagnosis of vasospastic diseases in vessels of different sizes into medical practice are of vital importance, and their relevance is undeniable. For the purposes of clinical need, the diagnostic techniques should be able to both provoke the potential spasm and quantitatively determine the severity of the disorder.

A wide range of experimental techniques has been developed in the past and adopted for diagnosis of the vasospastic disorders, including impedance plethysmography, ${ }^{3}$ Doppler ultrasound, ${ }^{4}$ nail capillaroscopy,${ }^{5}$ optical coherence tomography, ${ }^{6-8}$ diffusing-wave spectroscopy, ${ }^{9}$ electrical thermometry, ${ }^{10}$ thermal imaging diagnostics, ${ }^{11}$ and others. Nevertheless, these methods do not simultaneously meet all the criteria (noninvasive method, sensitive to spasm of peripheral minute vessels, steady operate during sporadic manifestation of the disease). Also, due to a number of other pragmatic issues and requirements (such as cost of the equipment, staff qualifications, and experience required for interpretation of the results), these methods are still more popular in the research rather than in clinical practice.

Thermography and thermal imaging methods provide valuable information regarding the presence of microcirculatory disorders indirectly, by assessing relative changes of temperature in the microvascular projection on the body surface, but they are rarely used in practice and are considered as auxiliaries. ${ }^{10,11}$

Laser Doppler flowmetry (LDF) exhibits an extremely high potential in the studies of blood microcirculation. ${ }^{12}$ LDF is based on the detection of Doppler shift of the laser light backscattered from moving red blood cells (RBC) in the microvasculature. Doppler shift is assessed quantitatively by an analysis of photocurrent raised at the photodetector, following a unified mathematical model suggested by Bonner and Nossal. ${ }^{12}$ The applied model links integral characteristics of photocurrent's power spectrum with the average concentration of RBCs within the measured volume in biological tissue (known also as a "sampling volume") and their average velocity. The measured LDF signal relates linearly to the velocity and concentration of RBCs in the sampling volume (SV) and is typically represented by the index of blood microcirculation (Im), also known as a "perfusion," assessed in conventional perfusion units (PU). With the LDF, it is possible not only to noninvasively evaluate the intensity of blood flow in the vascular bed but also to explore the collective response of rhythmic pulsations of blood. Indeed, as many other diagnostic modalities, the LDF approach has some limitations, including inability to provide absolute indexes of the blood flow and a relatively low level of repeatability of the measured experimental data. The latest is generally linked with the physiological heterogeneity of the probing tissues, as well as with the associated artifacts. ${ }^{13}$

To improve repeatability of the obtained LDF signal, typically, the standard physiological tests (such as occlusion, heat provocation, breath tests, and other) are executed. This makes it possible to obtain specific physiological information in the framework of the diagnostic protocol. To determine the onset of vasoconstriction in the deeper tissues (subcutaneous venous plexus), affecting most severely the environment heat exchange, ${ }^{14}$ the measurements of cutaneous temperature during step change

1083-3668/2017/\$25.00 @ 2017 SPIE 
of temperature of the surrounding medium with a high heat capacity (for example, water) can be used. It is anticipated that being prone to spasms of skin blood vessels is significantly higher for patients with vasospastic disorders in comparison to the temperature threshold of vasoconstriction for healthy volunteers.

An example of a functional physiological test that has an opposite effect on the state of vascular constriction is an occlusion test. During its practical implementation, the exceeding systolic pressure is created and maintained for a few minutes in the occlusive cuff on the limb. When cuff vascular clamping is removed, typically, a sharp increase of perfusion and temperature are observed with the subsequent restoration to normal. In physiology, this is explained as a vasodilation of vessels due to the accumulation of metabolic vasodilators in the occlusive period. ${ }^{15}$

In our study, a combined diagnostic procedure utilizing the occlusion test in the thermally stabilized medium (water) allows obtaining a better sensitivity and specificity of the diagnostic results. Cooling in water allows provoking the potential spasm, whereas the successive occlusion allows observing the ability of vascular regulation, with the excluding spasm state under the action of endogenous vasodilators. ${ }^{16}$ Therefore, in the framework of the proposed approach, a unified indicator of the normal/abnormal functional state of peripheral vessels, can be introduced. Thus, the promising area of use of the method in comparison with the abovementioned methods is noninvasive diagnostics of spasms of peripheral minute vessels in fingers with enhanced repeatability (using a special set of provocative tests).

\section{Materials and Method}

The diagnostics of vasospastic disorders has been tested on the skin of human fingers in vivo. The blood microcirculation was measured by the experimental system "LAKK-02" (SPE "LAZMA" Ltd., Russia). The developed in-house, multichannel thermometer was used for the low inertia thermometry (response time $0.25 \mathrm{~s}$ ), with the threshold sensitivity of $0.05^{\circ} \mathrm{C}$ and the compactness of primary temperature converters of $5.9 \times 2.1 \mathrm{~mm}$. The design of the combined LDF-thermometry probe is shown in Fig. 1.

The ability of the probe to register LDF signals in a particular vascular bed with the susceptibility to spasm has been verified using a Monte Carlo (MC)-based computational tool. ${ }^{17}$ The MC method is currently recognized as a "gold standard" for assessing optical radiation transfer in turbid tissue-like scattering media. The applied MC code is implemented utilizing the advantages of parallel computing on graphics processing units using compute unified device architecture. ${ }^{17}$ The model has a cloud-based HTML5 interface (available at Ref. 18) that provides access to various applications, including counting of SV and fluence rate distribution, imitation of skin spectra and skin color, OCT images modeling, and other. Thus, for the LDF probe shown in Fig. 1, an assessment of the SV has been performed. The feature of a particular probe's geometry, variations in spatial distribution of blood, melanin, index of blood oxygen saturation, hematocrit, volume fraction of water, as well as the numerical aperture and the detector positioning at the surface of the finger, were taken into account in a similar manner, as described earlier. ${ }^{19}$ The results of MC modeling are shown in Fig. 1 in the scale of a human finger. SV estimated by MC modeling for the LDF probe used in the experiment was found in a range $\sim 8 \mathrm{~mm}^{3}$, which is four times bigger compared to the standard optical fiber placed on the skin surface. The results of SV modeling also confirm that the LDF probe is highly sensitive to the variations of blood flows in the papillary dermis and upper blood net plexus, and is able see deeper into reticular dermis (see Fig. 1). Upper layers of dermis contain cutaneous thermoreceptors, which are localized at a depth ranging from 150 to $170 \mu \mathrm{m}$ for the cold receptors and from 300 to $600 \mu \mathrm{m}$ for the warmth receptors. ${ }^{14}$ In frame work of the modeling study, we assume that the vascular bed that is covered by the SV has no significant influence by the heat exchange with the external environment. On the contrary, unreachable by the probing light, the subcutaneous region contains the venous plexus, which strongly affects skin temperature and heat transfer from the skin to the external environment. At the same time, the blood flow in capillaries and the upper blood net plexus is also controlled by the sympathetic nervous system and depends on the needs of thermoregulation. Thus, the assessment of blood flow in the upper blood net plexus and in capillaries can act as an indirect estimate of the full response of the skin vascular system to temperature changes; it can also be used as an indicator of the onset of vasospasm vessels of the upper layers of the reticular dermis.

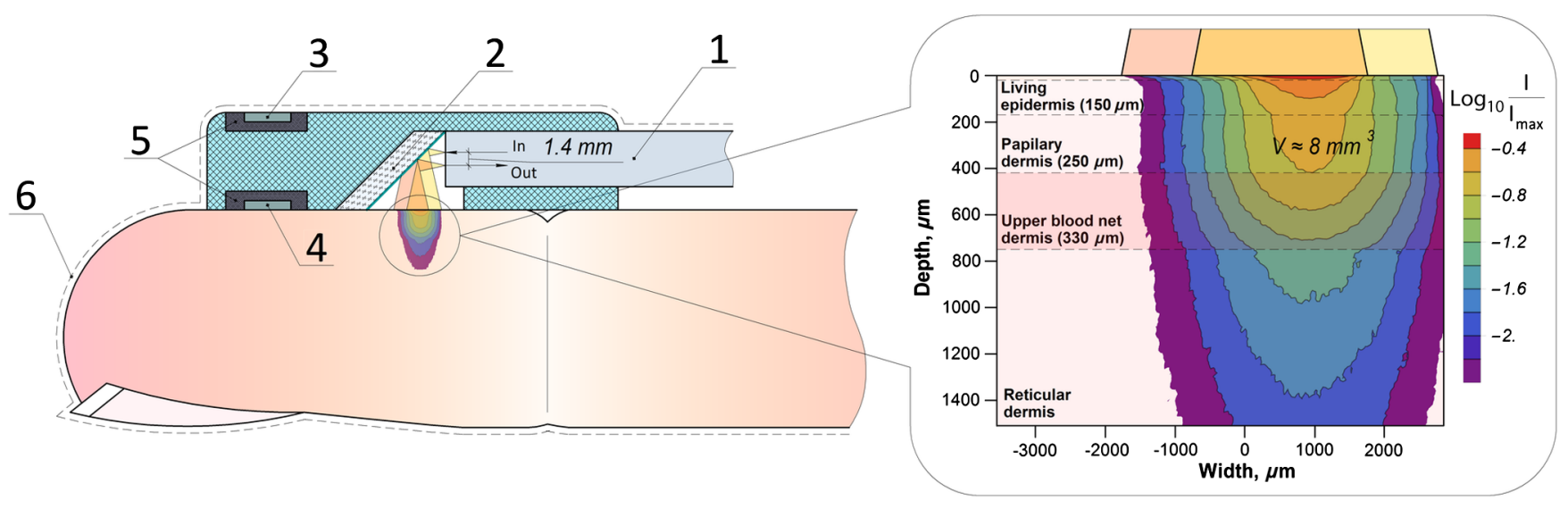

Fig. 1 Schematic presentation of the combined LDF-thermometry probe placed on the human finger: 1 , optical fiber probe; 2 , mirror; 3 , sensor for the measurements of surrounding temperature; 4 , sensor measuring skin temperature; 5, thermoinsulation; 6, waterproofing layer (latex). The insert (right) shows two-dimensional map of SV counted by MC modeling for the actual configuration of the optical probe used in the experiments (the wavelength of probing light is $1064 \mathrm{~nm}$ ). 


\section{Description of Diagnostic Approach}

Utilizing the probe described above, the measurements of cutaneous temperature and index of microcirculation were performed on the distal phalanx of the third finger of the right hand. Occlusion tests were performed with water baths at $25^{\circ} \mathrm{C}$ and a tonometer cuff with a pressure of 200 to $220 \mathrm{mmHg}$ for 3 min on the upper arm. In preocclusive (to set constant initial conditions of the study) and postocclusive (to relieve induced spasm) periods, the hand was immersed in a water bath at $42^{\circ} \mathrm{C}$. The use of the water temperature at $25^{\circ} \mathrm{C}$ meets both criteria to provide comfort for patients and a considerable dynamic range of skin postocclusive temperature. The patient sat in such a way that the forearm of his right hand was $20 \mathrm{~cm}$ below the level of the heart. Representative records of the cutaneous blood perfusion of healthy volunteers and rheumatological patients are shown in Fig. 2(a). Representative records of the skin temperature are shown in Fig. 2(b). The obtained results [see Fig. 2(a)] show that the signal recorded by LDF is affected by the ambient temperature, as well as by the imposition of occlusion. At the same time, in case of disorders, the relationships can be inadequate. For the example in Fig. 2(a), the perfusion level for rheumatological patient is not growing significantly after the period of occlusion.

It should be also pointed out that not only the ambient temperature, but also periods of imposition and removal of the occlusion affects the skin temperature [Fig. 2(b)]. The degree of increase in temperature $\left(T_{\max }\right)$ and perfusion $\left(I m_{\max }\right)$ after removal of the imposed occlusion characterizes the normal ability of the vasculature in ischemic conditions to secrete endogenous vasodilators, as well as to be triggered by them. Thus, the measurements of relative increase (with regard to baseline) of skin temperature and perfusion after removal of the occlusion provide information about the ability of the internal regulation of the vascular system to eliminate the state of spasm (provoked by moderate cooling in our case). Thus, LDF is highly sensitive to variations of blood flows in the superficial vessels in te dermis, whereas the record of skin temperature provides the diagnostics of the deeper vascular plexus, which affects the heat exchange of the skin much more strongly.

To assess the onset of angiospasm, we introduce the index of the temperature response

$\operatorname{ITR}=\frac{\left(T_{\max }-T_{\mathrm{cw}}\right) /\left(T_{\min }-T_{\mathrm{cw}}\right)}{V}$,

where $T_{\min }$ is the minimum temperature of the biological tissue during occlusion period, ${ }^{\circ} \mathrm{C} ; T_{\max }$ is the maximum temperature of the biological tissue during occlusion period, ${ }^{\circ} \mathrm{C} ; T_{\mathrm{cw}}$ is the minimum temperature of the water medium; and $V$ is the volume of the distal phalanx of the test finger, calculated as the volume of a semiellipsoid with semiaxes equal to half the height and width of a finger in the axial section of the interphalangeal joint between the proximal and distal phalanges and the length of the distal phalanx, in $\mathrm{cm}^{3}$.

Based on our experimental studies, we identified that the cutaneous thermometry can be supplemented by the LDF measurements. Thus, we apply the composite diagnostic criteria, which include both parameters provided by LDF and cutaneous thermometry. In this study, we have considered and tested several parameters describing a postocclusive reactive hyperemia, including time parameters of the transient processes. ${ }^{20,21}$ However, due to better predictive capability for the applied conditions, well known in the occlusion test diagnostic indicator,
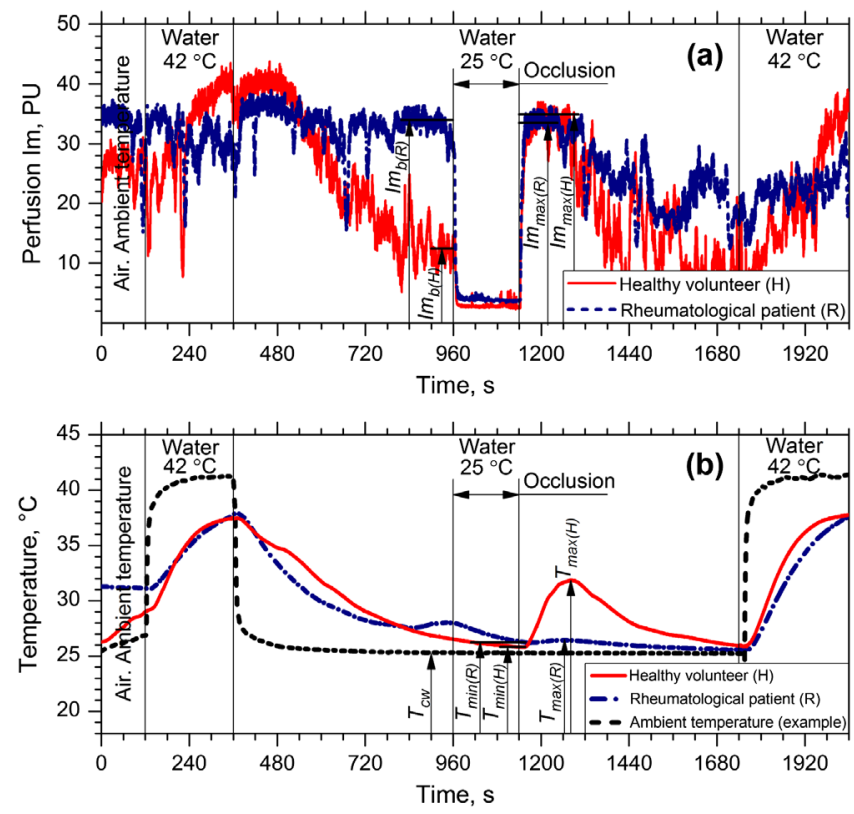

Fig. 2 An example of the results of a joint registration of the finger perfusion (a) (by LDF method) and cutaneous temperature (b) for healthy volunteer and rheumatological patient.

the so-called "blood flow reserve" (BFR) was selected as the primary LDF informative parameter. ${ }^{22}$

$\mathrm{BFR}=\frac{I m_{\max }}{I m_{b}} \cdot 100 \%$,

where $I m_{\max }$ is the average index of blood microcirculation in the first $60 \mathrm{~s}$ after occlusion, PU; $I m_{\text {base }}$ is the average index of blood microcirculation during $60 \mathrm{~s}$ before occlusion, PU.

The proposed approach was tested in the series of experiments on the group of healthy volunteers (13 men and 14 females, average age $23 \pm 5$ years) and the group of patients with rheumatoid arthritis and arthrosis with pronounced symptoms of vasospastic disorders ( 5 male and 36 females, average age $56 \pm 12$ years). In the framework of these feasibility studies, utilizing linear discriminant analysis, ${ }^{23}$ a phenomenological formula has been derived

$\left\{\begin{array}{l}\mathrm{BFR} \cdot 0.022+\mathrm{ITR} \cdot 1.61-3.70>0, \text { norma. } \\ \mathrm{BFR} \cdot 0.022+\mathrm{ITR} \cdot 1.61-3.70 \leq 0, \text { angiospastic violations }\end{array}\right.$

Equation 3 provides an opportunity to identify whether a patient has the angiospastic violations in their vascular system. The boxplot representing linear discriminant function scores for

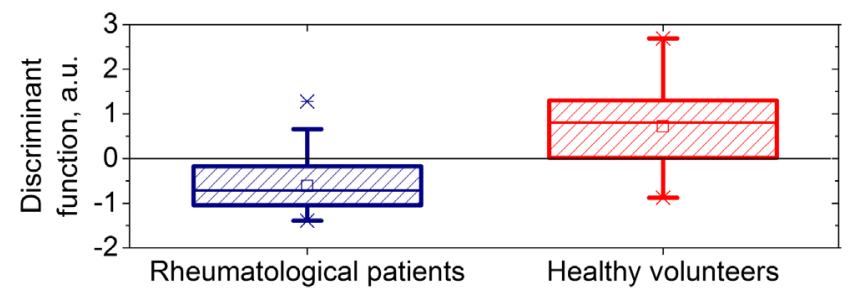

Fig. 3 Boxplot representing linear discriminant function scores for the groups of healthy volunteers and rheumatological patients. 


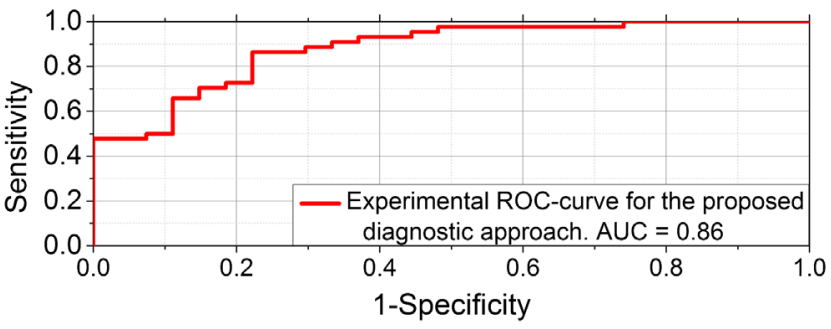

Fig. 4 Experimental ROC-curve for the proposed diagnostic approach.

the groups of healthy volunteers and rheumatological patients are shown in Fig. 3. The experimental ROC-curve of the technique is shown in Fig. 4. For the plot, the area under curve (AUC) equals 0.86 whereas the classifiers based only on ITR or $\mathrm{BFR}$ have $\mathrm{AUC}=0.76$ and $\mathrm{AUC}=0.79$, respectively. Thus, combined use of the parameters allows a significant increase in the sensitivity and specificity of diagnostics. Verification of Eq. (3) reveals the probability of a false-negative diagnosis result at the level of 0.13 (sensitivity 0.87 ) and a false-positive at 0.26 (specificity 0.74). ${ }^{24}$ All studies were approved by the Ethical Committee of the Regional Clinical Hospital. Thus, the proposed approach can be used for simple diagnostics of the functional state of peripheral vessels in vivo.

\section{Conclusions}

To sum up, utilizing the combined measurements of skin temperature and skin blood microcirculation during the occlusion test in the thermally stabilized medium, a new diagnostic approach for patient with angiospatic dysfunctions of the peripheral blood vasculature has been suggested. The approach is based on the assessment of reserve possibilities of blood flow using LDF and the reactivity of peripheral vessels located at a greater depth using the cutaneous thermometry method. The BFR and "index of the temperature response" were measured and used as the primarily parameters of the functional diagnostics of the peripheral vessels of skin. Utilizing these parameters, a simple phenomenological model to identify patients with the angiospastic violations in vascular system has been suggested, and it can be used concurrently with the existing diagnostic methods.

\section{Disclosures}

The authors declare the absence of any potential conflicts of interest relevant to this article.

\section{Acknowledgments}

The work was supported by a grant from the President of the Russian Federation for state support of young Russian scientists No. MK-7168.2016.8. The authors acknowledge the support of the Academy of Finland (Grant Nos: 296408 and 290596). Professor Meglinski also acknowledges partial support provided by the Russian Science Foundation project No. 15-14-10008.

\section{References}

1. J. A. Block and W. Sequeira, "Raynaud's phenomenon," Lancet 357(9273), 2042-2048 (2001).

2. M. D. Mayes, "Epidemiology of systemic sclerosis and related diseases," Curr. Opin. Rheumatol. 9, 557-561 (1997).
3. J. M. Porter, I. D. Swain, and P. G. Shakespeare, "Measurement of pulsatile limb and finger blood flow by electrical impedance plethysmography: criteria for the diagnosis of abnormal flow," J. Biomed. Eng. 9(4), 367-373 (1987)

4. E. de Miguel et al., "Ultrasound in rheumatology: where are we and where are we going?" Reumatología Clínica (English Edition) 10(1), 6-9 (2014).

5. M. E. Tavakol et al., "Nailfold capillaroscopy in rheumatic diseases: which parameters should be evaluated?" Biomed. Res. Int. 2015, 1-17 (2015).

6. A. Doronin and I. Meglinski, "Imaging of subcutaneous microcirculation vascular network by double correlation optical coherence tomography," Laser Photonics Rev. 7(5), 797-800 (2013).

7. M. Bonesi, S. G. Proskurin, and I. V. Meglinski, "Imaging of subcutaneous blood vessels and flow velocity profiles by optical coherence tomography," Laser Phys. 20(4), 891-899 (2010).

8. M. Bonesi et al., "Application of Doppler optical coherence tomography in rheological studies: blood flow and vessels mechanical properties evaluation," J. Innovative Opt. Health Sci. 02(04), 431-440 (2009).

9. I. Meglinski and V. V. Tuchin, "Diffusing wave spectroscopy: application for blood diagnostics," in Handbook of Coherent-Domain Optical Methods: Biomedical Diagnostics, Environmental Monitoring, and Materials Science, V. V. Tuchin, Ed., pp. 149-166, Springer, New York, New York (2013)

10. M. Naghavi et al., "New indices of endothelial function measured by digital thermal monitoring of vascular reactivity: data from 6084 patients registry," Int. J. Vasc. Med. 2016, 1348028 (2016).

11. A. Szentkuti, H. S. Kavanagh, and S. Grazio, "Infrared thermography and image analysis for biomedical use," Periodicum Biologorum 113(4), 385-392 (2011).

12. M. J. Leahy et al., "Principles and practice of the laser-Doppler perfusion technique," Technol. Health Care 7(2-3), 143-162 (1999).

13. A. V. Dunaev et al., "Investigating tissue respiration and skin microhaemocirculation under adaptive changes and the synchronization of blood flow and oxygen saturation rhythms," Physiol. Meas. 35(4), 607-621 (2014).

14. E. A. Arens and H. Zhang, "The skin's role in human thermoregulation and comfort," in Thermal and Moisture Transport in Fibrous Materials, N. Pan and P. Gibson, Eds., pp. 560-602, Woodhead Publishing Ltd. (2006)

15. M. G. Lopez et al., "Ischemic exercise hyperemia in the human forearm: reproducibility and roles of adenosine and nitric oxide," Eur. J. Appl. Physiol. 112(6), 2065-2072 (2012).

16. A. I. Zherebtsova et al., "Study of the functional state of peripheral vessels in fingers of rheumatological patients by means of laser Doppler flowmetry and cutaneous thermometry measurements," Proc. SPIE 9917, 99170M (2016)

17. A. Doronin and I. Meglinski, "Online object oriented Monte Carlo computational tool for the needs of biomedical optics," Biomed. Opt. Express 2(9), 2461-2469 (2011).

18. www.biophotonics.fi.

19. G. I. Petrov et al., "Human tissue color as viewed in high dynamic range optical spectral transmission measurements," Biomed. Opt. Express 3(9), 2154-2161 (2012).

20. G. Yu et al., "Time-dependent blood flow and oxygenation in human skeletal muscles measured with noninvasive near-infrared diffuse optical spectroscopies," J. Biomed. Opt. 10(2), 024027 (2005).

21. E. K. Englund et al., "Multiparametric assessment of vascular function in peripheral artery disease: dynamic measurement of skeletal muscle perfusion, blood-oxygen-level dependent dignal, and venous oxygen saturation," Circulation 8(4), e002673 (2015)

22. M. Roustit and J.-L. Cracowski, "Non-invasive assessment of skin microvascular function in humans: an insight into methods," Microcirculation 19(1), 47-64 (2012).

23. G. K. Demir and K. Ozmehmet, "Online local learning algorithms for linear discriminant analysis," Pattern Recognit. Lett. 26(4), 421-431 (2005).

24. C. R. Rao, J. Miller, and D. C. Rao, "Statistical learning in medical data analysis," in Handbook of Statistics: Epidemiology and Medical Statistics, C. R. Rao, J. Miller, and D. C. Rao, Eds., pp. 679-711, Elsevier, Netherlands (2007). 